\title{
Influência da convecção mista sobre a otimização geométrica de um arranjo triangular de cilindros em escoamentos laminares
}

Mixed Convective Influence over the Geometrical Optimization of a Triangular Arrangement of Cylinders in Laminar Flows

\author{
G. M. Barros ${ }^{1}$; D. M. do Carmo ${ }^{1}$; R. Cemin ${ }^{1}$; M. M. Galarça ${ }^{2}$; L. A. Isoldi ${ }^{1}$; \\ L.A.O. Rocha ${ }^{3}$, E. D. dos Santos ${ }^{1 *}$ \\ ${ }^{1}$ Escola de Engenharia, Universidade Federal do Rio Grande (FURG), 96201-900, Rio Grande - RS, Brasil \\ ${ }^{2}$ Instituto Federal de Educação, Ciência e Tecnologia do Rio Grande do Sul (IFRS), 90050-170, Rio Grande - RS, \\ Brasil. \\ ${ }^{3}$ Departamento de Engenharia Mecânica, Universidade Federal do Rio Grande do Sul (UFRGS), 90050-170, Porto \\ Alegre - RS, Brasil. \\ *elizaldosantos@furg.br
}

(Recebido em 15 de setembro de 2014; aceito em 29 de dezembro de 2014)

Este estudo numérico consiste em avaliar a forma geométrica de um arranjo triangular de cilindros submetidos a escoamentos transientes, bi-dimensionais, incompressíveis, laminares e com convecção mista por meios do Constructal Design. As simulações foram realizadas para escoamentos com números de Reynolds e Prandtl, de 100 e 0,71, respectivamente, avaliando-se três diferentes números de Richardson: $\mathrm{Ri}=0,1 ; 1,0$; e 10,0. As equações de conservação de massa, quantidade de movimento e energia foram resolvidas com o uso do método de volumes finitos (FVM - do inglês: Finite Volume Method). A área ocupada pelos cilindros é uma restrição geométrica do problema. O principal objetivo é avaliar a influência do Ri no efeito da razão $\mathrm{S}_{\mathrm{T}} / \mathrm{D}$ (passo transversal sobre o diâmetro) sobre o coeficiente de arrasto $\left(\mathrm{C}_{\mathrm{D}}\right)$ e o número de Nusselt $\left(\mathrm{Nu}_{\mathrm{D}}\right)$ entre os cilindros e o escoamento circundante, bem como obter as geometrias ótimas do problema multiobjetivo. Em todos os casos estudados a razão $S_{\mathrm{L}} / D$ (passo longitudinal sobre o diâmetro) foi mantida fixa, $S_{\mathrm{L}} / D=1,5$. Foi avaliado que a variação do mecanismo motriz do escoamento (causado pela variação do número de Richardson) não somente afetou as magnitudes de $\mathrm{C}_{\mathrm{D}}$ e $\mathrm{Nu}_{\mathrm{D}}$, mas também alterou o comportamento das camadas limite fluidodinâmica e térmica conduzindo a alterações no efeito da razão $S_{\mathrm{T}} / D$ sobre $\mathrm{C}_{\mathrm{D}}$ e $\mathrm{Nu}_{\mathrm{D}}$.

Palavras-chave: , Arranjo de Cilindros, Otimização Geométrica, Convecção Mista.

This numerical study is to evaluate the geometric shape of a triangular arrangement of cylinders subjected to transient flows, two-dimensional, incompressible, laminar and mixed convection by means of Constructal Design. The simulations were performed for Reynolds and Prandtl numbers, 100 and 0.71 , respectively, evaluating three different numbers of Richardson: $\mathrm{Ri}=$ $0.1 ; 1.0$; and 10.0. The mass conservation equations, momentum and energy were solved using the finite volume method (FVM - English: Finite Volume Method). The area occupied by the cylinder is a geometric constraint problem. The main objective is to evaluate the influence of $\mathrm{Ri}$ in the effect of the ratio $S_{\mathrm{T}} / D$ (cross step on the diameter) on the drag coefficient $\left(\mathrm{C}_{\mathrm{D}}\right)$ and the Nusselt number $\left(\mathrm{Nu}_{\mathrm{D}}\right)$ between the cylinders and the surrounding flow, and obtain the optimal geometry of the multi-objective problem. In all cases studied the ratio $S_{\mathrm{L}} / \mathrm{D}$ (on the longitudinal pitch diameter) was fixed $S_{\mathrm{L}} / D=1.5$. It was estimated that the variation of the driving mechanism of the flow (caused by the variation of Richardson number) have affected the $C_{D}$ magnitudes and $\mathrm{Nu}_{\mathrm{D}}$.

Keywords: Arrange of Cylinders, Geometrical Optimization, Mixed Convection

\section{INTRODUÇÃO}

Sistemas térmicos em problemas que envolvem interações entre um escoamento de fluido e superfícies são amplamente estudados e empregados em diversas áreas da engenharia. Os escoamentos externos sobre cilindros, em especial, representam de forma simplificada diversos problemas de engenharia como pontes, torres, conversores de energia eólica, condensadores, 
evaporadores, geradores de vapor, trocadores de calor multi-tubulares e outros. A maximização da transferência de calor entre as superfícies do arranjo e o escoamento circundante também tem sido alvo de estudos na literatura [1,2].

Muitos estudos são dedicados à avaliação da complexa fenomenologia envolvida nestes escoamentos, como a formação, o desprendimento e esteira de vórtices e seus padrões com relação a parâmetros do escoamento (números de Reynolds, Prandtl e Grashoff). Em Sahu et al. [3], foram analisados os efeitos dos números de Prandtl e Reynolds no comportamento fluidodinâmico e na transferência de calor para escoamento externo em um cilindro quadrado e em Catalano et al. [4] foi analisado o escoamento sobre um cilindro com elevados números de Reynolds.

Além dos estudos relacionados com a fenomenologia do escoamento, também é importante compreender a influência geométrica sobre o comportamento do escoamento, bem como, sobre a maximização dos desempenhos fluidodinâmico e térmico do escoamento. Nesse sentido, tem sido crescente o emprego do Constructal Design que é um método de avaliação geométrica baseado no princípio de restrições e objetivos e em um princípio físico de maximização do acesso ao fluxo. Esse princípio é denominado de Lei Constructal definida por "Para um sistema de tamanho finito persistir no tempo (sobreviver), o mesmo precisa evoluir sua forma e estrutura para providenciar um melhor acesso às correntes que fluem através dele" [5]. Em Bejan e Lorente [6], são apresentadas diversas aplicações da Lei Constructal para avaliação geométrica em problemas de transferência de calor.

O estudo de corpos submetidos a escoamentos sem o emprego do Constructal Design tem sido realizado na literatura. Por exemplo, em Song e Chang [7] são avaliados os padrões do escoamento e desprendimento de vórtices de um escoamento laminar sobre um par de cilindros posicionados transversalmente a direção principal do escoamento, i.e., com arranjo lado a lado (tandem). O comportamento dos vórtices também é estudado em Meinders e Hanjalic [8], dessa vez para um escoamento turbulento com convecção forçada sobre uma matriz de cubos. Um extenso trabalho de revisão bibliográfica é apresentado em Summer [9], com diversos estudos em escoamentos sobre um par de cilindros com arranjos alinhado e transversal. Neste trabalho, também foi avaliada a influência da relação $P / D$ (razão entre passo dos cilindros e diâmetros) sobre os parâmetros médios do escoamento como número de Strouhal $\left(\mathrm{St}_{\mathrm{D}}\right)$, coeficientes de arrasto $\left(C_{D}\right)$, sustentação $\left(C_{L}\right)$ e número de Nusselt $\left(\mathrm{Nu}_{D}\right)$. Em Alam e Zhou [10], foi feita uma simulação numérica sobre escoamento sobre 2 cilindros em tandem.

Com relação à otimização da convecção de calor com aplicação da Lei Constructal é possível mencionar o trabalho onde foi estudada a minimização da resistência térmica em micro-canais de resfriamento [11]. Em Bello-Ochende et al.[12], foi avaliado o efeito sobre a transferência de calor em dutos utilizando entradas corrugadas e em Bello-Ochende e Bejan [13] foi feito um estudo sobre a otimização da troca térmica em um escoamento laminar com convecção natural transversal a um arranjo de cilindros de múltiplas escalas. Neste estudo considerou-se um domínio bidimensional. Em Silva e Bejan [14] é apresentado um estudo de escoamentos com convecção forçada sobre arranjos de placas planas com várias escalas de comprimento. Posteriormente, foi realizado um estudo sobre a convecção forçada e natural em um arranjo transversal de dutos [15]. Neste trabalho foi analisada a corrente de escoamento nos lados externo e interno. Foi realizada a simulação de escoamentos sobre arranjos de cilindros de múltiplas escalas com rotação e os resultados demonstraram que a taxa de transferência de calor aumenta conforme a complexidade geométrica do arranjo aumenta [16].

Com relação ao problema multi-objetivo, em Rocha et al. [17] foram estudados numericamente escoamentos com convecção forçada, transientes, bidimensionais, incompressíveis e turbulentos sobre um par de cilindros. Em todos os casos foi considerado um escoamento com $\mathrm{Re}_{\mathrm{D}}=22.000$ e $\operatorname{Pr}=0,71$. O principal objetivo foi avaliar o melhor ângulo entre o par de cilindros e a direção principal do escoamento que conduziria a maior troca térmica, bem como, minimizaria a força de arrasto entre os cilindros e o escoamento circundante. Como resultado foi demonstrado que os melhores arranjos para o escoamento em questão situam-se numa faixa de 45 a 60 graus considerando-se os dois objetivos propostos no trabalho. Em Razera e Cardoso [18] foi realizado um estudo numérico similar para um 
escoamento laminar com convecção forçada $\left(\operatorname{Re}_{\mathrm{D}}=100\right.$ e $\left.\operatorname{Pr}=0,71\right)$ sobre um arranjo triangular de cilindros avaliando-se a influência dos passos longitudinal e transversal.

No presente trabalho a Lei Constructal é aplicada para otimizar as relações $S_{\mathrm{T}} / D$ (passo transversal sobre o diâmetro do cilindro) para um $S_{\mathrm{L}} / D$ (passo longitudinal sobre o diâmetro do cilindro) fixo $\left(S_{\mathrm{L}} / D=1,5\right)$ em arranjo triangular de três cilindros imersos em escoamentos com convecção mista, laminar e transiente. O principal objetivo aqui é determinar o arranjo ótimo que maximiza o coeficiente de transferência de calor e minimiza o coeficiente de arrasto, para diferentes números de Richardson $\left(\mathrm{Ri}=\mathrm{Gr}_{\mathrm{D}} / \mathrm{Re}_{\mathrm{D}}{ }^{2}=0,1 ; 1,0\right.$ e 10,0$)$, bem como, avaliar a influência do número de Richardson sobre a geometria ótima e efeito da geometria no comportamento fluidodinâmico e térmico do escoamento. Dessa forma, são simulados escoamentos dominados pela convecção forçada $(\mathrm{Ri}=0,1)$ em equilíbrio entre a convecção forçada e mista $(\mathrm{Ri}=1.0)$ e dominados pela convecção natural $(\mathrm{Ri}=10,0)$. Vale destacar que, os números de Grashoff e Reynolds são dados por: $\mathrm{Gr}_{\mathrm{D}}=g \beta \Delta T H^{3} / v^{2}$ e $\operatorname{Re}_{\mathrm{D}}=u_{\infty} D / \mathrm{v}$, respectivamente. Para a obtenção dos campos de velocidades, pressão e temperaturas as equações de conservação de massa, quantidade de movimento e energia são resolvidas numericamente através do método de volumes finitos $[19,20]$, mais precisamente empregando o código de dinâmica dos fluidos computacional FLUENT ${ }^{\mathrm{TM}}$ [21].

\section{MODELAGEM MATEMÁTICA E NUMÉRICA}

A modelagem dos escoamentos incompressíveis, transientes com convecção mista no regime laminar é baseada na solução das equações de conservação em conjunto com as condições de contorno e iniciais do problema. As equações de conservação de massa, quantidade de movimento e energia são dados, respectivamente, por [22]:

$$
\begin{array}{lr}
\frac{\partial v_{j}}{\partial x_{j}}=0 & (j=1 \text { e } 2) \mathrm{em} t \times \Omega \\
\frac{\partial}{\partial t}\left(v_{i}\right)+\frac{\partial}{\partial x_{j}}\left(v_{i} v_{j}\right)+\frac{1}{\rho} \frac{\partial P}{\partial x_{j}} \delta_{i j}-\frac{\partial}{\partial x_{j}}\left\{v\left(\frac{\partial v_{i}}{\partial x_{j}}+\frac{\partial v_{j}}{\partial x_{i}}\right)\right\}+\rho g_{i} \beta\left(T-T_{0}\right)=0 & (i, j, k=1 \text { e } 2) \mathrm{em} t \times \Omega \\
\frac{\partial T}{\partial t}+\frac{\partial}{\partial x_{j}}\left(v_{j} T\right)-\frac{\partial}{\partial x_{j}}\left\{\alpha \frac{\partial T}{\partial x_{j}}\right\}-q^{\prime \prime \prime}=0 & (j=1 \text { e } 2) \mathrm{em} t \times \Omega
\end{array}
$$

onde $\rho$ é a massa específica do fluido $\left(\mathrm{kg} / \mathrm{m}^{3}\right) ; \mu$ é a viscosidade dinâmica do fluido $(\mathrm{kg} /(\mathrm{ms})) ; v$ é a viscosidade cinemática do fluido $\left(\mathrm{m}^{2} / \mathrm{s}\right) ; \alpha$ é a difusividade térmica $\left(\mathrm{m}^{2} / \mathrm{s}\right) ; v_{\mathrm{i}}$ é a velocidade do fluido na direção $\mathrm{i}, \mathrm{i}=1$ e $2(\mathrm{~m} / \mathrm{s}) ; x_{\mathrm{i}}$ corresponde a coordenada espacial na direção $\mathrm{i}, \mathrm{i}=1 \mathrm{e}$ $2(\mathrm{~m}) ; P$ é a pressão $\left(\mathrm{N} / \mathrm{m}^{2}\right) ; T$ é a temperatura $\left({ }^{\circ} \mathrm{C}\right.$ ou $\left.\mathrm{K}\right) ; \delta_{\mathrm{ij}}$ é o operador delta de Kronecker; $g_{\mathrm{i}}$ é a aceleração da gravidade na direção i; $\beta$ é o coeficiente de expansão térmica $\left(\mathrm{K}^{-1}\right) ; \Omega$ é o domínio espacial (m); $t$ representa o domínio de tempo (s), e q"' é o termo fonte de energia, que para os casos simulados no presente trabalho é nulo.

As Eqs. (1) - (3) são resolvidas através de um software comercial de dinâmica dos fluidos computacional (CFD - do inglês: Computational Fluid Dynamics) que é baseado no método de volumes finitos (FVM - do inglês: Finite Volume Method) [19 - 20]. Em todas as simulações foram empregados volumes finitos retangulares. O solver é baseado na pressão, o acoplamento pressão-velocidade foi realizado com o uso do algoritmo SIMPLE e para o tratamento dos termos advectivos foi empregado o esquema de interpolação Upwind de segunda ordem para as equações de conservação de quantidade de movimento e energia. Além disso, os cálculos foram 
considerados convergidos quando os resíduos para as equações de conservação de massa, quantidade de movimento e energia entre duas iterações consecutivas foram menores do que 10-6, 10-6 e 10-8, respectivamente. Maiores detalhes relacionados com o FVM podem ser encontrados em Patankar [19] e Versteeg e Malalasekera [20].

As simulações numéricas foram realizadas usando um computador com dois processadores dual-core Intel com 3.6 GHz de clock e 24GB de memória RAM. Para a paralelização foi utilizada a biblioteca de passagem de mensagem (MPI - do inglês: Message Passing Interface). O tempo de processamento para cada simulação foi de aproximadamente $7,20 \times 10^{2} \mathrm{~s}$.

A independência de malha seguiu conforme obtido por Razera e Cardoso [18], bem como os resultados para convecção forçada pura.

\section{DESCRIÇÃO DO PROBLEMA}

Para o problema deste estudo, foi considerado um escoamento sobre um arranjo triangular de cilindros com o mesmo diâmetro $D=0,1 \mathrm{~m}$. O domínio computacional possui as seguintes dimensões: $L=35,0 D=3.5 \mathrm{~m}, H=15,0 D=1,5 \mathrm{~m}, L_{1}=8 D=0,8 \mathrm{~m}$. A Figura 1 apresenta $\mathrm{o}$ domínio do escoamento, onde é possível observar também a variável que será variada no presente trabalho: $S_{\mathrm{L}} / D$ (passo longitudinal pelo diâmetro dos cilindros). Com relação ao comportamento fluidodinâmico do escoamento, o mesmo é gerado pela imposição de um perfil de velocidades constante u $\infty$ (velocidade na corrente livre) na superfície de entrada e também pela força de campo oriunda da diferença de temperaturas entre os cilindros e o escoamento circundante. $\mathrm{O}$ problema é resolvido por similaridade de forma a garantir $\operatorname{Re}_{\mathrm{D}}$ e $\mathrm{Gr}_{\mathrm{D}}$. As superfícies dos cilindros possuem a condição de contorno de não-deslizamento e impermeabilidade $\left(u_{\mathrm{w}}=0 \mathrm{~m} / \mathrm{s}\right)$. As superfícies inferior e superior possuem uma condição de simetria e a superfície lateral direita possui uma condição de contorno de pressão manométrica nula. A convecção natural é causada pela ação do campo gravitacional, que neste trabalho é considerada por uma força de campo (hipótese de Boussinesq). A aceleração do campo gravitacional também é ilustrada na Figura 1. Para o problema térmico, o aquecimento do fluido é causado pela diferença de temperatura entre a corrente livre do escoamento $\left(T_{\infty}=20^{\circ} \mathrm{C}\right)$ que é constante e imposta na superfície de entrada e a temperatura dos cilindros $\left(T_{\mathrm{w}}=30^{\circ} \mathrm{C}\right)$.

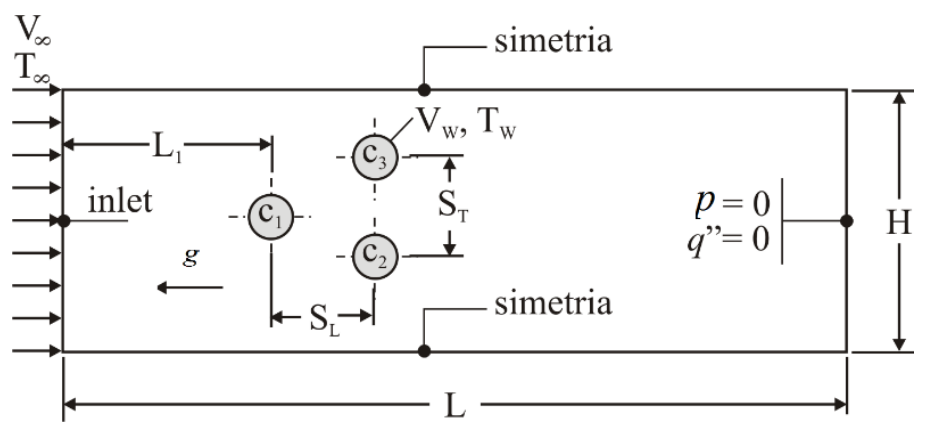

Figura 1: Domínio computacional do escoamento sobre arranjo triangular de cilindros com $\operatorname{Re}_{D}=$ 100 e $\operatorname{Pr}=0.71$.

Para a otimização geométrica do problema é empregado o método Constructal Design [5-6]. Constructal Design é um método baseado no princípio de objetivos e restrições (locais e globais) sendo o método usado para aplicação da Lei Constructal, já comentada anteriormente. Para o problema analisado, foram definidas como restrições geométricas do problema: 1) a área ocupada pelos três cilindros, que é a mesma em todos os casos estudados $\left(A_{\mathrm{t}}=A_{\mathrm{c} 1}+A_{\mathrm{c} 2}+A_{\mathrm{c} 3}\right)$, 2) os diâmetros dos cilindros são iguais $\left(D_{1}=D_{2}=D_{3}=D\right)$ e 3) o espaço ocupado pelo par de cilindros à jusante do primeiro cilindro não pode ser superior a altura do domínio computacional empregado $\left(S_{\mathrm{T}}+D<H\right)$.

O processo de otimização é dividido em duas etapas, conforme ilustra a Figura 2. No primeiro passo, a geometria é otimizada pela variação do grau de liberdade $S_{\mathrm{T}} / D$ mantendo-se 
fixo o parâmetro $S_{\mathrm{L}} / D$. A geometria que conduzir ao maior valor de número de Nusselt $\left(\mathrm{Nu}_{\mathrm{D}}\right)$ será a ótima para o problema térmico e a que conduzir ao menor valor para o coeficiente de arrasto $\left(\mathrm{C}_{\mathrm{D}}\right)$ será a geometria ótima para o problema fluidodinâmico. O parâmetro $S_{\mathrm{T}} / D$ correspondente será uma vez otimizado, $\left(S_{\mathrm{T}} / D\right)_{\mathrm{o}}$. No segundo passo, o mesmo processo é repetido para vários valores de $S_{\mathrm{L}} / D$. No presente estudo, o parâmetro $S_{\mathrm{L}} / D$ é mantido constante $\left(S_{\mathrm{L}} / D=1,5\right)$, enquanto o parâmetro $S_{\mathrm{T}} / D$ é variado na seguinte faixa $\left(1,5 \leq S_{\mathrm{T}} / D \leq 5,0\right)$. Esse processo é repetido para cada número de Richardson estudado, neste caso três valores: $\mathrm{Ri}=$ $\mathrm{Gr}_{\mathrm{D}} / \operatorname{Re}_{\mathrm{D}}{ }^{2}=0,1 ; 1,0$ e 10,0 .

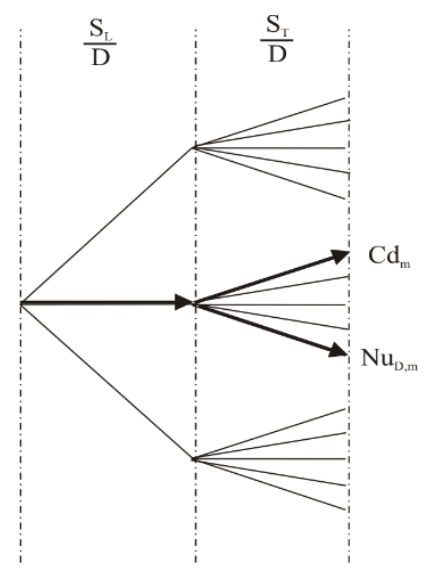

Figura 2: Diagrama ilustrando o processo de otimização para um problema com dois graus de liberdade

\section{RESULTADOS E DISCUSSÕES}

A Figura 3 apresenta o efeito da razão $S_{\mathrm{T}} / D$ sobre o coeficiente de arrasto $\left(\mathrm{C}_{\mathrm{D}}\right)$, Figura $3(\mathrm{a})$, e sobre o número de Nusselt $\left(\mathrm{NuD}\right.$ ), Figura $3(\mathrm{~b})$, para $S_{\mathrm{L}} / D=1,5$ e $\mathrm{Ri}=0,1$ (caso com convecção forçada dominante). De uma forma geral, é observado que o coeficiente de arrasto aumenta à medida que o passo transversal também aumenta para todos os cilindros. Contudo, para o cilindro a montante no escoamento $\left(\mathrm{C}_{1}\right)$ a variação de magnitude com $S_{\mathrm{T}} / D$ é mais considerável (de $\mathrm{C}_{\mathrm{D}}=1,0$ em $S_{\mathrm{T}} / D=1,5$ para $\mathrm{C}_{\mathrm{D}}=1,8$ em $S_{\mathrm{T}} / D=5,0$ ) do que para os cilindros $\mathrm{C}_{2}$ e $\mathrm{C}_{3}$, que estão a jusante do cilindro $\mathrm{C}_{1}$. Esse comportamento está principalmente relacionado com a diferença de pressão entre a região frontal e posterior do primeiro cilindro. Para o caso $S_{\mathrm{T}} / D=$ 1,5 , os cilindros estão muito próximos e não há uma formação de uma região de baixa pressão em uma região posterior próxima ao cilindro $\mathrm{C}_{1}$. Conforme há um aumento da razão $S_{\mathrm{T}} / D$ os cilindros tendem a encarar quase que individualmente a corrente livre do escoamento que incide sobre os cilindros, conduzindo também a formação de uma região de pressão negativa similar ao observado em escoamentos sobre apenas um cilindro. Com relação aos cilindros $\mathrm{C}_{2}$ e $\mathrm{C}_{3}$, para o caso onde os cilindros estão mais próximos $\left(S_{\mathrm{T}} / D=1,5\right)$ é observada uma pequena assimetria no padrão de recirculação do escoamento após os cilindros $\mathrm{C}_{2}$ e $\mathrm{C}_{3}$, conduzindo a forças de arrasto também diferentes. Conforme a razão $S_{\mathrm{T}} / D$ aumenta o escoamento tende a ser mais simétrico e os coeficientes de arrasto dos cilindros $\mathrm{C}_{2}$ e $\mathrm{C}_{3}$ convergem para um mesmo valor. Com relação ao número de Nusselt, Figura 3(b), pode se observar um comportamento análogo ao obtido para o coeficiente de arrasto nos três cilindros. Para o cilindro $\mathrm{C}_{1}$, observa-se um $\mathrm{Nu}_{\mathrm{D}}$ pequeno para as menores relações de $S_{\mathrm{T}} / D$ e conforme o afastamento dos cilindros aumenta há um aumento do $\mathrm{Nu}_{\mathrm{D}}$ nesse cilindro. Também pode ser notado que há uma convergência no número de Nusselt para os cilindros para as razões mais afastadas de $S_{\mathrm{T}} / D$. Esse comportamento está relacionado a não interação das camadas limites térmicas para estes casos. Vale destacar ainda que para o caso com $\mathrm{Ri}=0,1$ há uma pequena alteração na magnitude do $\mathrm{C}_{\mathrm{D}}$ e $\mathrm{Nu}_{\mathrm{D}}$ em comparação com o caso de convecção forçada [18]. Apesar disso, o comportamento do $\mathrm{C}_{\mathrm{D}}$ e $\mathrm{Nu}_{\mathrm{D}}$ em função de $S_{\mathrm{T}} / D$ para $S_{\mathrm{L}} / D=1,5$ são semelhantes aos observados para o caso de convecção forçada.Uma conclusão deve ser apresentada. 

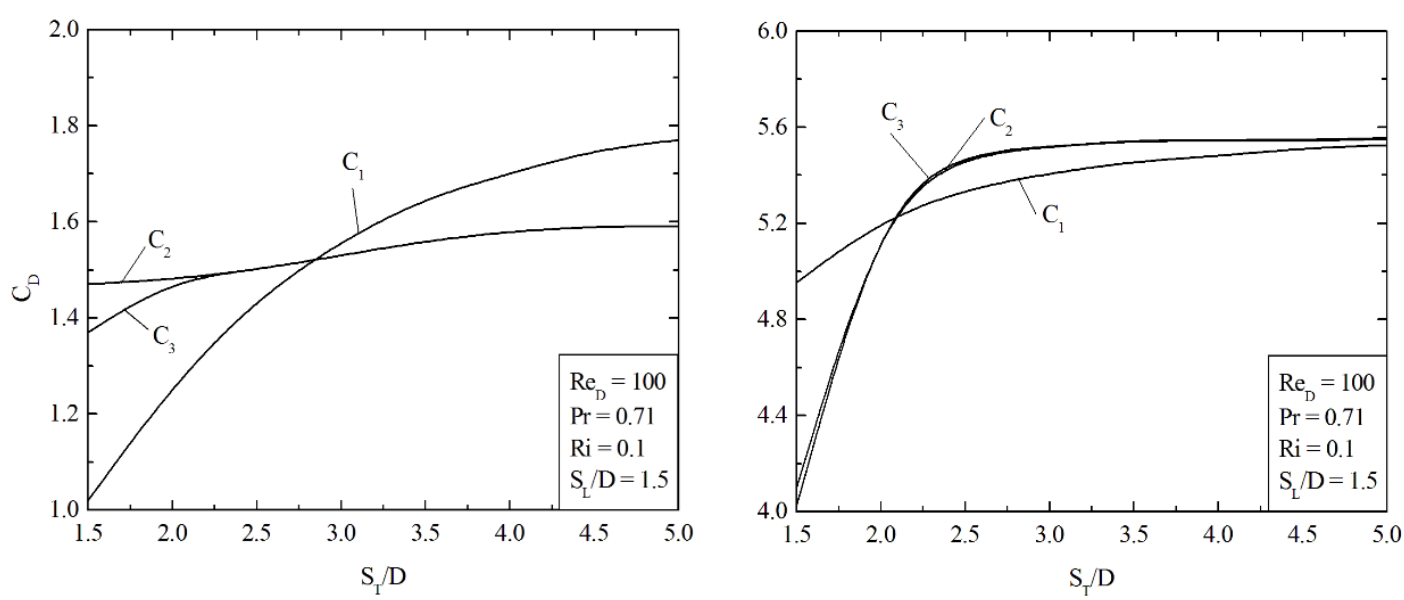

Figura 3: Efeito do parâmetro $S_{\mathrm{T}} / \mathrm{D}$ para um escoamento a $\mathrm{Re}_{\mathrm{D}}=100, \mathrm{Pr}=0.71$ e $\mathrm{Ri}=0.1$ sobre os seguintes parâmetros em cada cilindro: a) coeficiente de arrasto $\left(\mathrm{C}_{\mathrm{D}}\right)$, b) número de $\mathrm{Nusselt}\left(\mathrm{Nu}_{\mathrm{D}}\right)$.

A Figura 4 apresenta o efeito da razão $S_{\mathrm{T}} / D$ sobre o coeficiente de arrasto $\left(\mathrm{C}_{\mathrm{D}}\right)$, Figura $4(\mathrm{a})$, e sobre o número de Nusselt $\left(\mathrm{Nu}_{\mathrm{D}}\right.$ ), Figura $4(\mathrm{~b})$, para $S_{\mathrm{L}} / D=1,5$ e $\mathrm{Ri}=10,0$ (caso com convecção natural dominante). De uma forma geral, as magnitudes dos coeficientes de arrasto para todos os cilindros $\left(\mathrm{C}_{1}, \mathrm{C}_{2}\right.$ e $\left.\mathrm{C}_{3}\right)$ aumentaram em comparação com o caso a $\mathrm{Ri}=0,1$, o que era esperado por tratar-se da combinação da convecção forçada com a convecção natural, i.e., é adicionada uma força de campo ao escoamento com convecção forçada. Além disso, essa força aumenta com o aumento do número de Grashoff. A Figura 4 também ilustra que o efeito de $S_{\mathrm{T}} / D$ sobre $C_{D}$, Figura 4(a), e $\mathrm{Nu}_{\mathrm{D}}$, Figura 4(b), são diferentes dos observados para os casos com convecção forçada e $\mathrm{Ri}=0,1$. Para o coeficiente de arrasto, o $\mathrm{C}_{\mathrm{D}}$ para o cilindro $\mathrm{C}_{1}$ possui um crescimento nas menores razões de $S_{\mathrm{T}} / D$ até um máximo em $S_{\mathrm{T}} / D=2,5$ e após este ponto há uma diminuição na magnitude de $C_{D}$, o que não ocorre para o caso com $R i=0,1$. Para os cilindros $C_{2}$ e $C_{3} o$ comportamento é ainda mais diferente do observado para $\mathrm{Ri}=0,1$. Para as menores razões de $S_{\mathrm{T}} / D$ há um decréscimo de $\mathrm{C}_{\mathrm{D}}$ até um ponto de mínimo em $S_{\mathrm{T}} / D=3,0$. Posteriormente, a magnitude de $\mathrm{C}_{\mathrm{D}}$ torna a crescer novamente. Para as menores razões de $S_{\mathrm{T}} / D$ ocorre a formação de uma única pluma ascendente, que leva a formação de uma região de baixa pressão após os cilindros $\mathrm{C}_{2}$ e $\mathrm{C}_{3}$. Enquanto que, para as maiores razões $S_{\mathrm{T}} / D$ a formação da pluma é similar à observada para apenas um cilindro. Vale destacar que estes resultados não indicam para o arranjo uma região de ótimo, visto que quando o $C_{D}$ nos cilindros $C_{2}$ e $C_{3}$ são mínimos o observado para o cilindro $\mathrm{C}_{1}$ está em uma região máxima. Com relação ao número de Nusselt $\left(\mathrm{Nu}_{\mathrm{D}}\right)$, como era esperado, a associação da convecção forçada com a natural com um maior número de Grashoff conduz a uma taxa de transferência de calor mais intensa em todos os cilindros. Também é observado que o efeito da razão $S_{\mathrm{T}} / D$ é bastante diferente do que o obtido para casos de convecção forçada [18]. Neste caso são observadas razões ótimas intermediárias para cada cilindro, o que não foi obtido nos casos onde a convecção forçada é dominante. Neste caso $(\mathrm{Ri}=10,0)$, para as menores razões de $S_{\mathrm{T}} / D$, há interferência entre as camadas limite térmicas de cada cilindro, prejudicando a troca térmica entre os cilindros e o escoamento. No extremo superior, o desempenho térmico também é minimizado devido ao decréscimo da quantidade de movimento entre os cilindros. 

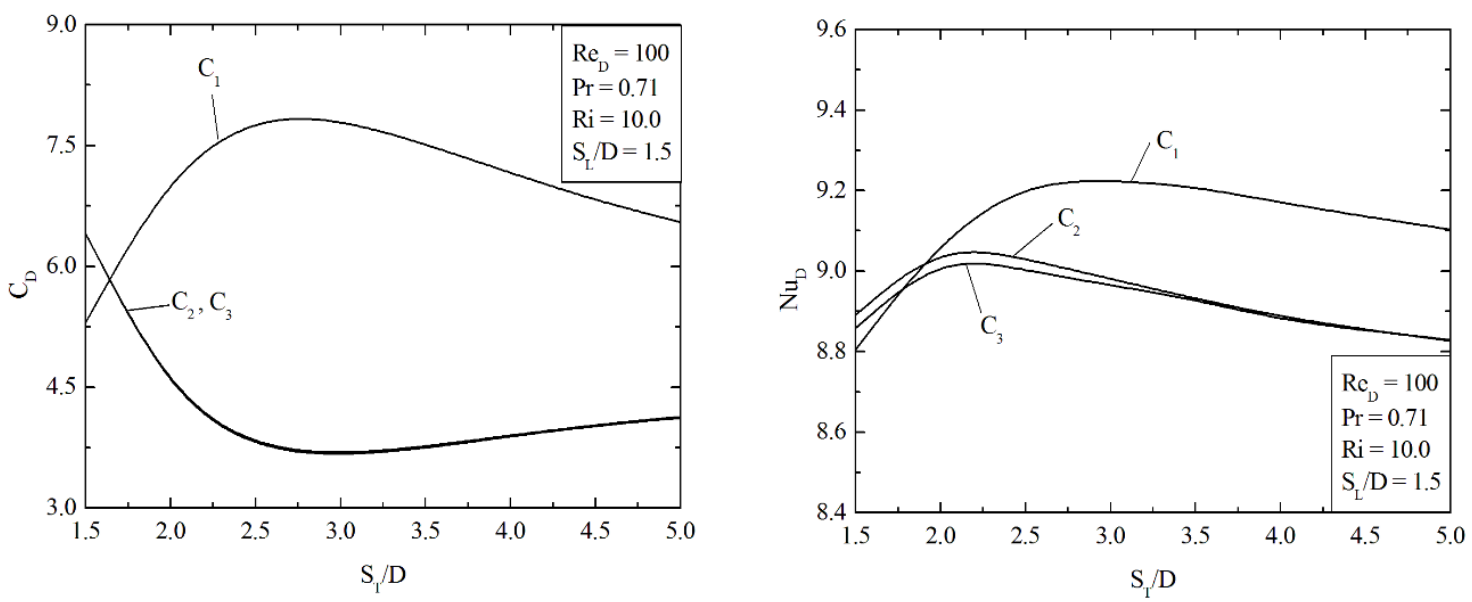

Figura 4: Efeito do parâmetro $S_{T} / D$ para um escoamento a $\operatorname{Re}_{D}=100, \operatorname{Pr}=0,71$ e Ri $=0,1$ sobre os seguintes parâmetros em cada cilindro: a) coeficiente de arrasto $\left(C_{D}\right)$, b) número de Nusselt $\left(N u_{D}\right)$.

Para verificar o efeito de $S_{\mathrm{T}} / D$ sobre o $\mathrm{C}_{\mathrm{D}}$ e $\mathrm{Nu}_{\mathrm{D}}$ no arranjo dos cilindros foi realizada uma média entre os valores obtidos em cada um dos cilindros para cada caso de Ri investigado e também para o caso com convecção forçada. A Figura 5 ilustra o efeito de $S_{\mathrm{T}} / D$ sobre o coeficiente de arrasto médio nos três cilindros $\left(\mathrm{C}_{1}, \mathrm{C}_{2}\right.$ e $\left.\mathrm{C}_{3}\right)$ para os casos com convecção forçada e mista com $\mathrm{Ri}=0,1 ; 1,0$ e 10,0. Pode ser observado que para os casos com convecção forçada predominante a razão uma vez otimizada de $S_{\mathrm{T}} / D$ é obtida quando os cilindros estão o mais próximo possíveis, i.e., $\left(S_{\mathrm{T}} / D\right)_{\mathrm{o}}=1,5$. Esse fato está principalmente relacionado pois o arrasto devido a diferença no cilindro $\mathrm{C}_{1}$ é significativamente reduzido. Para o caso $\mathrm{Ri}=1,0 \mathrm{o}$ $\mathrm{C}_{\mathrm{D}}$ é quase insensível a razão $S_{\mathrm{T}} / D$. Apesar das diferenças serem pequenas, o melhor desempenho foi obtido para a razão $\left(S_{\mathrm{T}} / D\right)_{\mathrm{o}}=2,0$. Quando a magnitude de Ri cresce ainda mais para $\mathrm{Ri}=10,0$ e a convecção mista passa a ser o mecanismo motriz dominante $\mathrm{o}$ comportamento de $\mathrm{C}_{\mathrm{D}}$ em função de $S_{\mathrm{T}} / D$ é oposto ao obtido para os casos onde a convecção forçada é dominante. Neste caso, a magnitude de $\mathrm{C}_{\mathrm{D}}$ diminui com o aumento da razão $S_{\mathrm{T}} / D$ e a geometria ótima é encontrada para a geometria com cilindros mais afastados, $\left(S_{\mathrm{T}} / D\right)_{\mathrm{o}}=5,5$.

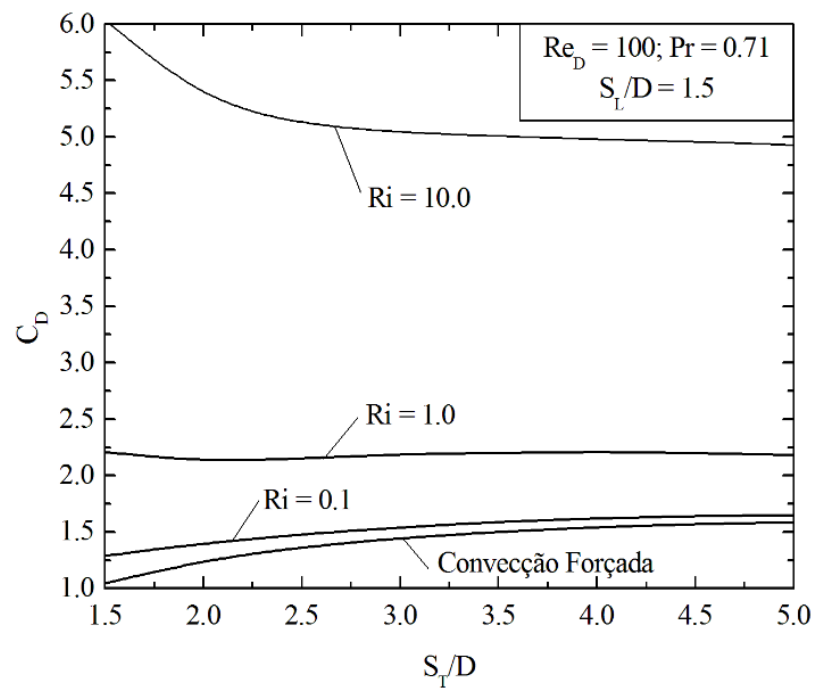

Figura 5: Efeito do parâmetro $S_{T} / D$ sobre o $C_{D}$ médio do arranjo para diferentes números de Richardson e $S_{L} / D=1,5$.

$\mathrm{O}$ mesmo procedimento realizado para $\mathrm{C}_{\mathrm{D}}$ na Figura 5 é repetido para o número de $\mathrm{Nu}_{\mathrm{D}} \cdot \mathrm{A}$ Figura 6 ilustra o número de $\mathrm{Nu}_{\mathrm{D}}$ médio para o arranjo de cilindros para os casos com convecção forçada e com $\mathrm{Ri}=0,1 ; 1,0$ e 10,0. Da mesma forma vista para o coeficiente de arrasto, o número de Nusselt $\left(\mathrm{Nu}_{\mathrm{D}}\right)$ para os casos onde a convecção forçada é dominante aumenta com o aumento da razão $S_{\mathrm{T}} / D$. A geometria ótima para os casos onde a convecção 
forçada é dominante é obtida para $\left(S_{\mathrm{T}} / D\right)_{\mathrm{o}}=5,0$. Conforme o número de Ri cresce observa-se que a geometria ótima passa a ser obtida para razões intermediárias de $S_{\mathrm{T}} / D$, para $\mathrm{Ri}=1,0$ a geometria ótima é obtida para $S_{\mathrm{T}} / D=3,0$, enquanto que para $\mathrm{Ri}=10.0$ a geometria ótima passa a ser obtida para $S_{\mathrm{T}} / D=2,5$. Também pode ser notado que com o crescimento de Ri as diferenças entre o desempenho obtido com a geometria ótima e o limite inferior diminuiram.

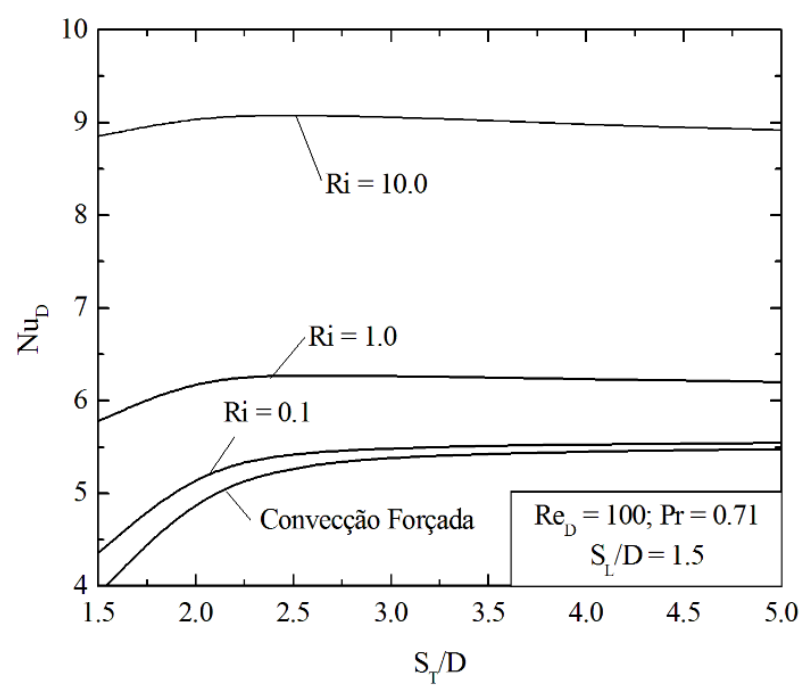

Figura 6: Efeito do parâmetro $S_{T} / D$ sobre o $N u_{D}$ médio do arranjo para diferentes números de Richardson e $S_{L} / D=1,5$.

Os resultados ótimos e suas respectivas geometrias obtidos na Figura 5 para os vários números de Richardson avaliados são sumarizados na Figura 7. A Figura 7 ilustra o efeito do número de Richarson sobre o coeficiente de arrasto uma vez minimizado $\left(\mathrm{C}_{\mathrm{D}, \mathrm{m}}\right)$ e sobre a razão ótima de $S_{\mathrm{T}} / D,\left(S_{\mathrm{T}} / D\right)_{\mathrm{o}}$. Os resultados indicam que ha um crescimento quase linear do $\mathrm{C}_{\mathrm{D}, \mathrm{m}}$ com o aumento do número de Richardson no escoamento. Além disso, a geometria ótima, $\left(S_{\mathrm{T}} / D\right)_{\mathrm{o}}$, também cresce em uma relação linear com o aumento do número de Richardson, ou seja, a geometria se adapta ao tipo de escoamento para minimizar a resistência ao escoamento.

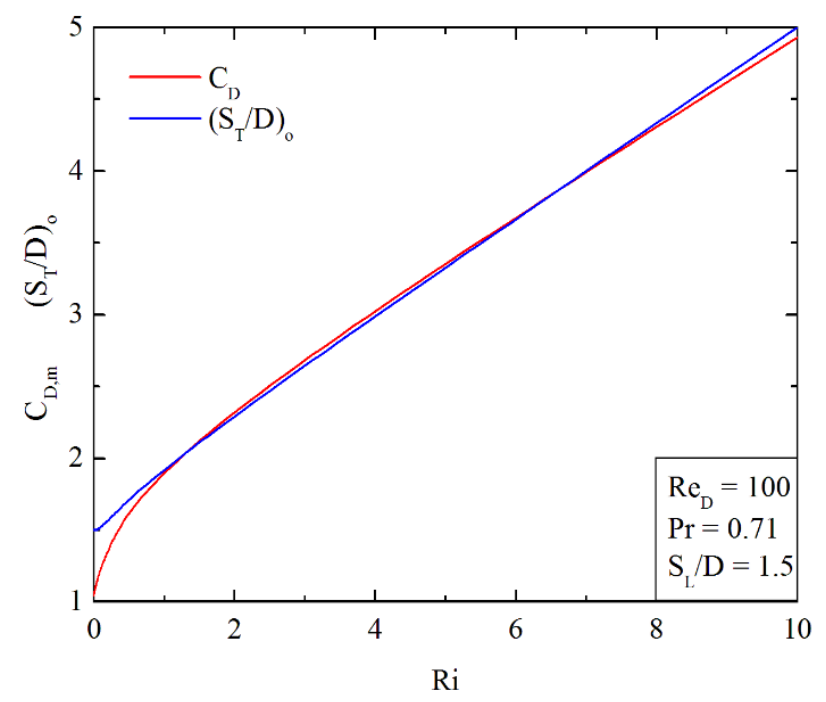

Figura 7: Efeito do número de Richardson sobre o $C D_{m}$ e $\left(S_{T} / D\right)_{o}$.

O processo análogo foi realizado quanto ao problema térmico. Nesse sentido os resultados ótimos e suas respectivas geometrias obtidos na Figura 6 para vários números de Richardson são sumarizados na Figura 8. A Figura 8 ilustra o efeito do número de Richardson sobre o número de Nusselt uma vez maximizado $\left(\mathrm{Nu}_{\mathrm{D}, \mathrm{m}}\right)$ e sobre a razão ótima de $S_{\mathrm{T}} / D,\left(S_{\mathrm{T}} / D\right)_{\mathrm{o}}$. Semelhantemente ao observado para o problema fluidodinâmico, o número de Nusselt uma vez 
maximizado aumenta com o crescimento do número de Richardson. Contudo, o comportamento da geometria foi diferente do observado para o problema fluidodinâmico. Na Figura 8 pode ser visto que a geometria ótima sofre um rápido decréscimo da maior razão $\left(S_{\mathrm{T}} / D\right)_{0}=5,0$ para o caso de convecção forçada para $\left(S_{\mathrm{T}} / D\right)_{\mathrm{o}} \cong 3,5 \mathrm{em} \mathrm{Ri}=1,0$. A partir de $\mathrm{Ri}=5,0$ a geometria ótima estabiliza em $\left(S_{\mathrm{T}} / D\right)_{\mathrm{o}}=2,5$. Para baixos Ri (convecção forçada dominante) as camadas limites térmicas em cada cilindro são espessas e a interação entre elas deixa de ocorrer para elevadas razões de $S_{\mathrm{T}} / D$, enquanto que com o aumento do número de Richardson a pluma ascendente se forma em uma região estreita próxima do cilindro e as interações entre camadas limite térmicas se limita a relações pequenas de $S_{\mathrm{T}} / \mathrm{D}$. A razão $\left(S_{\mathrm{T}} / D\right)_{\mathrm{o}}=2,5$ se localiza em uma região onde não há mais interação entre a camada limite térmica e onde há um aumento da quantidade de movimento na região entre os cilindros oriundo da corrente livre que é imposta na entrada do domínio. Para razões maiores de $S_{\mathrm{T}} / D$ não há interação entre as camadas limites térmicas, mas a quantidade de movimento é mais baixa, enquanto que para as menores razões de $S_{\mathrm{T}} / D$ a interação das camadas limite térmicas é elevada.

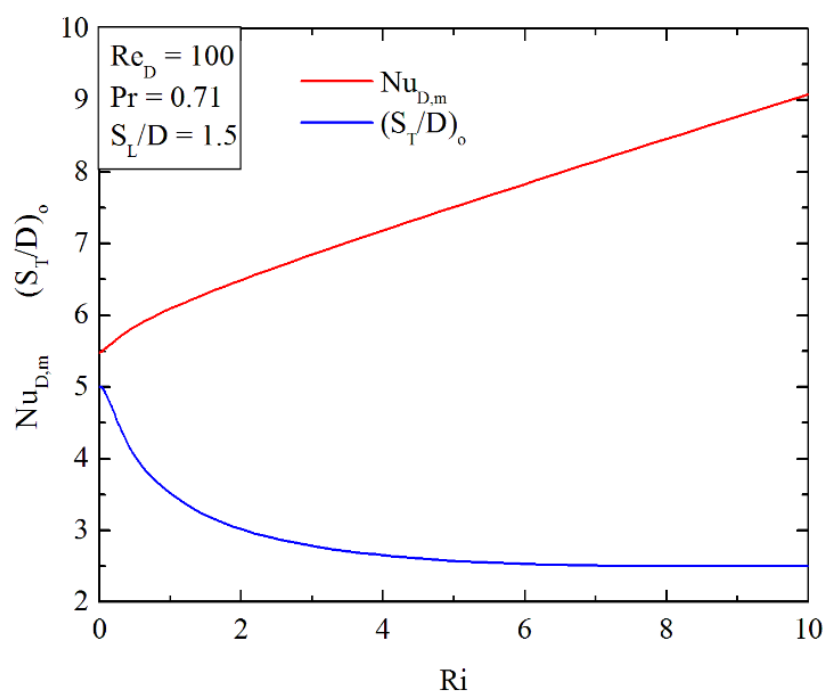

Figura 8: Efeito do número de Richardson sobre $N u_{D, m}$ e $\left(S_{T} / D\right)_{o}$.

\section{CONCLUSÕES}

Foi realizado um estudo numérico para avaliar a forma geométrica de um arranjo triangular de cilindros submetidos a escoamentos laminares com convecção mista empregando o Constructal Design. As simulações foram realizadas para escoamentos com números de Reynolds e Prandtl de $\operatorname{Re}_{\mathrm{D}}=100$ e $\operatorname{Pr}=0,71$ avaliando-se três diferentes números de Richardson: $\mathrm{Ri}=0,1 ; 1,0$ e 10,0. A equações de conservação de quantidade de movimento e energia foram resolvidas com o uso do método de volumes finitos (FVM). O principal objetivo foi avaliar a influência do Ri no efeito da razão $S_{\mathrm{T}} / D$ (passo transversal sobre o diâmetro) sobre o coeficiente de arrasto $\left(\mathrm{C}_{\mathrm{D}}\right)$ e o número de Nusselt $\left(\mathrm{Nu}_{\mathrm{D}}\right)$ entre os cilindros e o escoamento circundante, bem como obter as geometrias ótimas do problema multiobjetivo.

Os resultados mostraram que o aumento do número de Richardson conduziu a um crescimento das magnitudes de $\mathrm{C}_{\mathrm{D}}$ e $\mathrm{Nu}_{\mathrm{D}}$. Além disso, houve uma mudança significativa do comportamento das camadas limites fluidodinâmica e térmica com o aumento de Ri que alterou o efeito da razão $S_{\mathrm{T}} / D$ sobre $\mathrm{C}_{\mathrm{D}}$ e $\mathrm{Nu}_{\mathrm{D}}$. Por exemplo, para $\mathrm{Ri}=0,1$ a geometria ótima no problema térmico é obtida para as maiores razões de $S_{\mathrm{T}} / D$, enquanto que para $\mathrm{Ri}=10,0$ uma razão intermediária de $S_{\mathrm{T}} / D$ passa a conduzir aos melhore resultados. Em outras palavras, não há uma geometria ótima universal que conduza ao melhor desempenho térmico. A geometria se adapta ao mecanismo motriz imposto ao arranjo de cilindros. 


\section{AGRADECIMENTOS}

Os professores L. A. Isoldi, L. A. O. Rocha e E. D. dos Santos agradecem ao CNPq pelo apoio financeiro. O estudante G. M. Barros agradece a CAPES pela bolsa de mestrado.

\section{REFERÊNCIAS BIBLIOGRÁFICAS}

1. Çengel YA, Cimbala JM. Fluid Mechanics: fundamentals and application. New York. McGraw-Hill; 2006.

2. Rajani BN, Kandasamy A, Majumdar S. Numerical Simulation of Laminar Flow Past a Circular Cylinder. Appl. Math. Model 2009;33(3):1228-47.

3. Sahu AK, Chhabra RP, Eswaran V. Effects of Reynolds and Prandtl Numbers on Heat Transfer from a Square Cylinder in the Unsteady Flow Regime. Int. J. Heat Mass Transfer 2009;52(3-4):839-850.

4. Catalano P, Wang M, Iaccarino G, Moin P. Numerical Simulation of the Flow around a Circular Cylinder at High Reynolds Numbers. Int. J. Heat Fluid Flow 2003;24(4):463-469; doi: 10.1016/S0142-727X(03)00061-4.

5. Bejan A. Shape and Structure, from Engineering to Nature. Cambridge University Press, Cambridge, UK; 2000.

6. Bejan A, Lorente S. Design with Constructal Theory. Wiley, Hoboken; 2008.

7. Song C-J, Chang K-S. Heat Transfer and Interactive Buoyant Vortex Sheeding by a Pair of Circular Cylinders in Transverse Arrangement. Int. J. Heat Mass Transfer 1991;34:1347-1354.

8. Meinders ER, Hanjalic K. Vortex Structure and Heat Transfer in Turbulent Flow over a WallMounted Matrix of Cubes. Int. J. Heat Fluid Flow 1999;20:255-267.

9. Summer D. Two Circular Cylinders in Cross-Flow: a Review. J. Fluid Struct. 2010;26:849-899; doi: 10.1016/j.jfluidstructs.2010.07.001.

10. Alam MM, Zhou Y. Strouhal Numbers, Forces and Flow Structures around Two Tandem Cylinders of Different Diameters. J. Fluid Struct. 2008;24:505-526; doi: 10.1016/j.jfluidstructs.2007.10.001.

11. Bello-Ochende T, Lienbenberg L, Malan AG, Bejan A, Meyer JP. Constructal conjugate heat transfer in three-dimensional cooling channels. J. Enhanc. Heat Transfer 2007;14:279-293.

12. Bello-Ochende T, Lienbenberg L, Malan AG, Bejan A, Meyer JP. Constructal ducts with wrinkled entrances. Int. J. Heat Mass Transfer 2009;52(15 - 16):3628-3633.

13. Bello-Ochende T, Bejan A. Constructal Multi-Scale Cylinders with Natural Convection. Int. J. Heat Mass Transfer 2005;48:4300-4306.

14. Silva AK, Bejan A. Constructal Multi-Scale Structure for Maximal Heat Transfer Density in Natural Convection. Int. J. Heat Fluid Flow 2005;26:34-44.

15. Kim Y, Lorente S, Bejan A. Constructal Multi-Tube Configuration for Natural and Forced Convection in Cross-Flow. Int. J. Heat Mass Transfer 2010;53(23-24):5121-5128; doi: 10.1016/j.ijheatmasstransfer.2010.07.053.

16. Bello-Ochende T, Meyer JP, Ogunronbi OI. Constructal Multiscale Cylinders Rotating in Cross-Flow. Int. J. Heat Mass Transfer 2011;54(11-12):2568- 2577; doi: 10.1016/j.ijheatmasstransfer.2011.02.004.

17. Rocha LAO, Gomes MN, Porte AF, Galarça MM, Acunha Jr IC, da Silva FMV, Isoldi LA, Dos Santos ED. Constructal Design of Turbulent Forced Convective Flows over a Pair of Circular cylinders. In: Constructal Law Conference, Nanjing. Proceedings of Constructal Law Conference, 2013; 1:174-184.

18. Razera AL, Cardoso FR. Otimização Geométrica do Arranjo de Tubos em Escoamentos por Convecção Forçada, Trabalho de Conclusão de Curso em Engenharia Mecânica, Universidade Federal do Rio Grande, 2013.

19. Patankar SV. Numerical heat transfer and fluid flow, McGraw Hill, New York, USA; 1980.

20. Versteeg HK, Malalasekera W. An introduction to computational fluid dynamics - the finite volume method, Pearson, England; 2007.

21. Fluent (version 13.0), ANSYS, Inc.; 2010.

22. Bejan A. Convection Heat Transfer, John Wiley, Durham, USA; 2004. 